\title{
Analysis of PM Magnetization Field Effects on the Unbalanced Magnetic Forces due to Rotor Eccentricity in BLDC Motors
}

\author{
S. Mahdiuon-Rad \\ Department of Electrical \\ Engineering, Sahand \\ University of Technology, \\ Tabriz, Iran \\ S_mahdiuonrad@sut.ac.ir
}

\author{
S. R. Mousavi-Aghdam \\ Department of Electrical \& \\ Computer Engineering \\ University of Tabriz, \\ Tabriz, Iran \\ Rmousavi@tabrizu.ac.ir
}

\author{
M. Reza Feyzi \\ Department of Electrical \& \\ Computer Engineering \\ University of Tabriz \\ Tabriz, Iran \\ Feyzi@tabrizu.ac.ir
}

\author{
M. B. B. Sharifian \\ Department of Electrical \& \\ Computer Engineering \\ University of Tabriz \\ Tabriz, Iran \\ Sharifian@tabrizu.ac.ir
}

\begin{abstract}
This paper investigates both static and dynamic eccentricities in single phase brushless DC (BLDC) motors and analyzes the effect of the PM magnetization field on unbalanced magnetic forces acting on the rotor. Three common types of PM magnetization field patterns including radial, parallel and sinusoidal magnetizations are considered. In both static and dynamic eccentricities, harmonic components of the unbalanced magnetic forces on the rotor are extracted and analyzed. Based on simulation results, the magnetization fields that produce the lowest and highest unbalanced magnetic forces are determined in rotor eccentricity conditions.
\end{abstract}

Keywords- finite element method; single phase brushless DC (BLDC) motor; static and dynamic eccentricity; unbalanced magnetic force

\section{INTRODUCTION}

Rotor eccentricity occurs when an unbalanced air gap exists between the stator and the rotor. Therefore, an unbalanced magnetic flux density between the rotor and the stator is occurred, which is the main contributor to magnetically induced vibration and noise [1].

Rotor eccentricity in a motor can be divided into three categories: static eccentricity, dynamic eccentricity, and their combination [2]. Unbalanced magnetic force exists as long as there is eccentricity between the rotor and the stator, because a portion of the stator is closer to the permanent magnet of the rotor, thus generating a net attraction force acting on the rotor [3]. Unbalanced magnetic force is also important because it has an exhausting effect on the bearings and also generates noise and vibration. On the other side, when eccentricity becomes large, the resulting unbalanced radial forces can cause stator-torotor rub, and this can result to stator and motor damage [4].

In a surface mounted permanent magnet motor, the permanent magnets act as air gaps (magnetically) between the iron in the stator and rotor. Small changes in actual air gap length have a negligible impress on the motor effective air gap. This suggests that surface mounted PM motors may be less sensitive to rotor eccentricities than induction motors, and would be good candidates for applications where noise and vibration are significant [5]. Therefore, a detailed study of unbalanced magnetic forces due to rotor eccentricity in such motors would be very useful.

It should be noted that in the design of permanent magnet motors for high-precision applications, it is sometimes necessary to have a detailed analysis of the effect of rotor eccentricity [6]. In this paper PM magnetization field effects on the unbalanced magnetic forces due to both static and dynamic eccentricities in single phase BLDC motors are analyzed.

\section{STATIC AND DYNAMIC ECCENTRICITY}

The external rotor permanent magnet motor with rotor eccentricity is schematically shown in Figure 1.

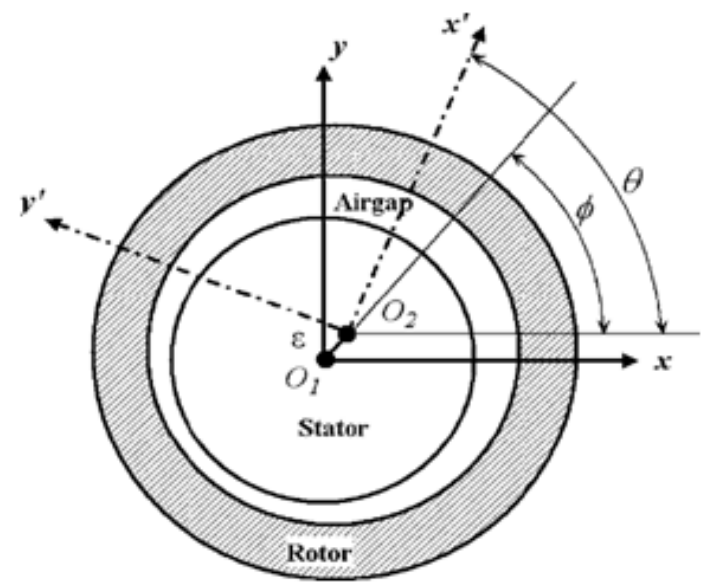

Fig. 1. Schematic of the external rotor PM motor with rotor eccentricity.

The radial distance between the stator axis $(O 1)$ and rotor axis $(\mathrm{O} 2)$ is defined as the eccentricity of the rotor and is denoted [2] by

$$
\varepsilon=e . g
$$


where $e$ is the eccentricity ratio and $g$ is the nominal air gap length. The eccentricity ratio has the following limit [2]:

$$
0 \leq e \leq 1
$$

Static eccentricity with which the rotor is displaced from the stator center but is still turning upon its own axis $O 2$ can be modeled by assuming $\varepsilon$ and $\phi$ as constants [2], that the position of the minimal radial air gap length is fixed in space and that there is a steady pull in one direction. This makes the unbalanced magnetic force difficult to be detected unless special equipment is used, which is impractical for motors in service.

Typical causes of static eccentricity include bearing wear, out of tolerance manufacturing and incorrect positioning of the rotor or the stator at assembly stage. Dynamic eccentricity is where the rotor does not rotate on its own axis but does rotate on the stator axis and the center of the rotor is not at the center of rotation so that the point of minimum air gap rotates with rotor speed. This means that dynamic eccentricity is a function of space and time and can be treated by considering $\varepsilon$ and $\phi$ as functions of time and position. In this case the rotor center rotates around the stator center. Dynamic eccentricity produces a radial magnetic pull that rotates at the mechanical speed of the motor and acts directly on the rotor. This makes the unbalanced magnetic force easier to detect by vibration or current monitoring [4]. Dynamic eccentricity could be caused by a bent shaft, mechanical resonances at critical speeds, bearing wear, out of tolerance manufacturing, using fluid dynamic or aerodynamic bearings and misalignment of bearings.

\section{DIFFERENT TYPES OF PM MAGNETIZATION FIELDS}

The single phase BLDC motor geometry considered for simulation is shown in Figure 2 [7]. Different PM magnetization field patterns can give different air gap field distributions. Therefore, the effect of PM magnetization field on the unbalanced magnetic forces due to rotor eccentricity of PM motor is investigated in this paper. For surface mounted permanent magnets, three common types of PM magnetization field patterns including radial, parallel and sinusoidal are considered as shown in Figure 3. Motor specifications are listed in Table I.

TABLE I. MOTOR SPECIFICATIONS.

\begin{tabular}{|c|c|}
\hline Stator inner diameter & $18 \mathrm{~mm}$ \\
\hline Stator outer diameter & $42 \mathrm{~mm}$ \\
\hline Rotor inner diameter & $48 \mathrm{~mm}$ \\
\hline Coil turns & 72 \\
\hline Number of pole & 4 \\
\hline PM material & ferrite \\
\hline Rotor outer diameter & $60 \mathrm{~mm}$ \\
\hline Permanent magnet thickness & $4 \mathrm{~mm}$ \\
\hline $\mathbf{H}_{\mathrm{C}}$ & $200 \mathrm{KA} / \mathrm{m}$ \\
\hline $\mathbf{B}_{\mathbf{r}}$ & $0.25 \mathrm{~T}$ \\
\hline Winding type & Concentrated winding \\
\hline Rated speed & $6000 \mathrm{rpm}$ \\
\hline
\end{tabular}

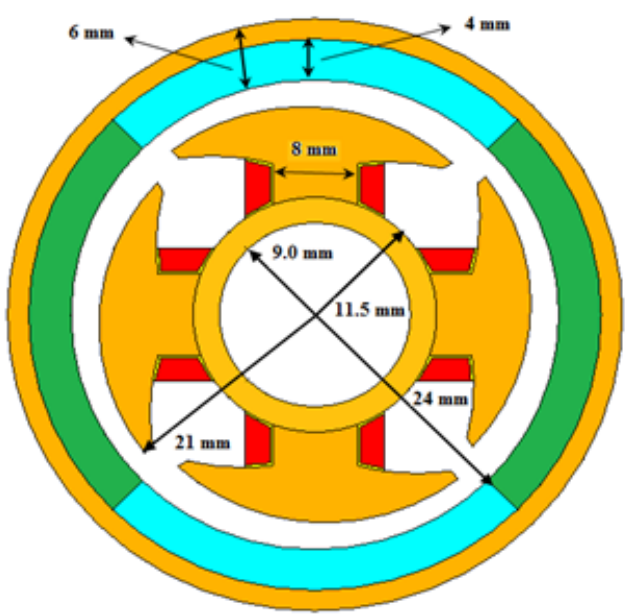

Fig. 2. Single phase BLDC motor geometry.
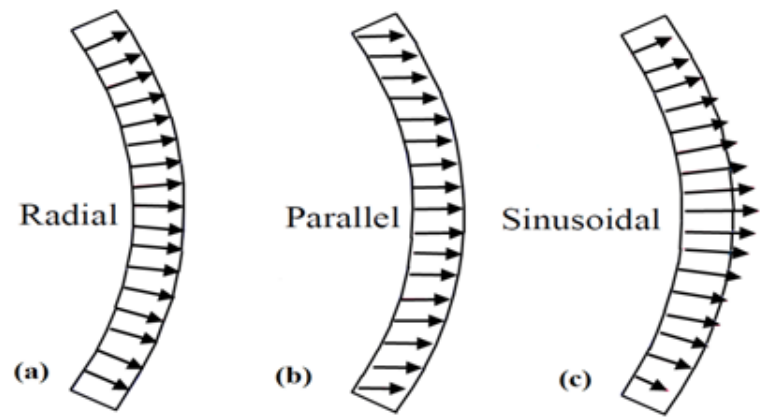

Fig. 3. (a) radial magnetization (b) parallel magnetization (c) sinusoidal magnetization.

\section{STATIC ECCENTRICITY ANALYSIS AND RESULTS}

Unbalanced magnetic forces with eccentricity ratios of 0.3 , 0.6 and 0.9 which are also known as radial magnetic forces, have been compared for various eccentricities. Figure 4 shows the 2-D finite-element model of the single phase BLDC motor used for the calculation of the unbalanced magnetic forces. The radial magnetic force on the rotor is calculated using Maxwell stress tensor at every 3 degrees of rotor position period and a total number of 120 calculations are needed for the simulation of a complete revolution. Radial magnetic forces on the rotor due to different eccentricities for parallel, radial and sinusoidal magnetization are shown in Figures 5-7.

In the static eccentricity analysis, the radial magnetic force directions are fixed in a complete revolution and magnitudes of these forces fluctuate between maximum and minimum values. It is shown that the average unbalanced magnetic force on the rotor and the force ripple increase with the eccentricity ratio. Harmonic components of radial magnetic forces on the rotor for various eccentricity ratios are compared and shown in Figure 8. Harmonic components of radial magnetic force increase with the eccentricity ratio and 4th, 8th and 12th 
harmonic components are greater than other components as shown in Figure 8. As mentioned earlier, in the case of static eccentricity, the position of the minimal radial air gap length is fixed in space. In other words, the part of the stator which is closer to the magnet is stationary and experiences different magnet polarity $N P$ times per revolution. Where $N P$ is the number of magnet poles. This leads to $i \times N P$ harmonics where $i$ is a positive integer.

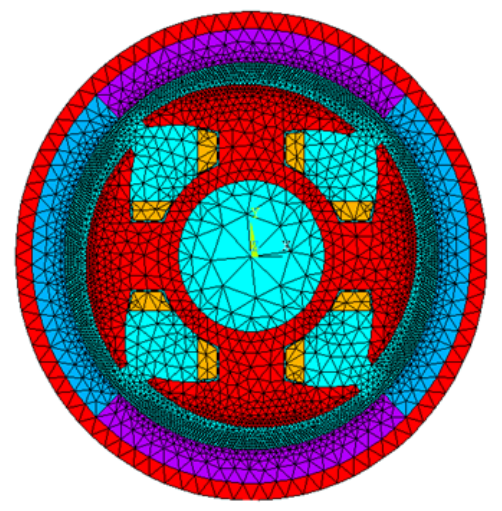

Fig. 4. 2-D finite-element model of the single phase BLDC motor.

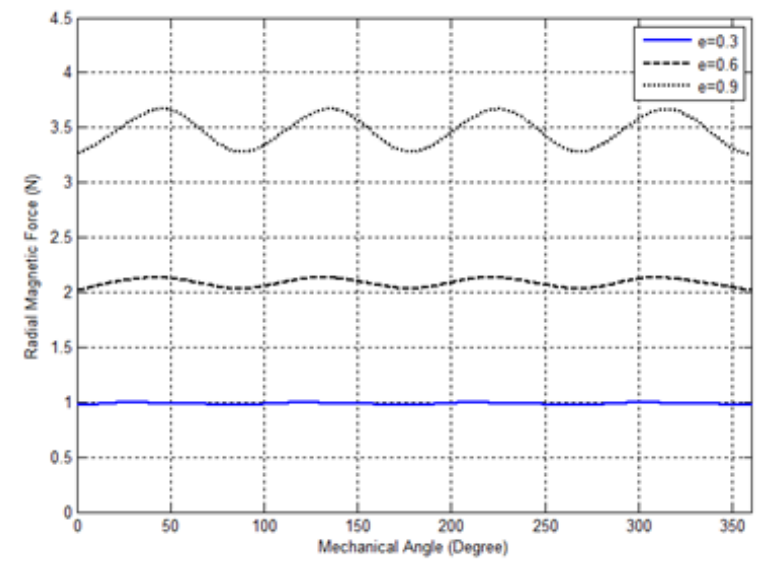

Fig. 5. Radial magnetic forces on the rotor for parallel magnetization.

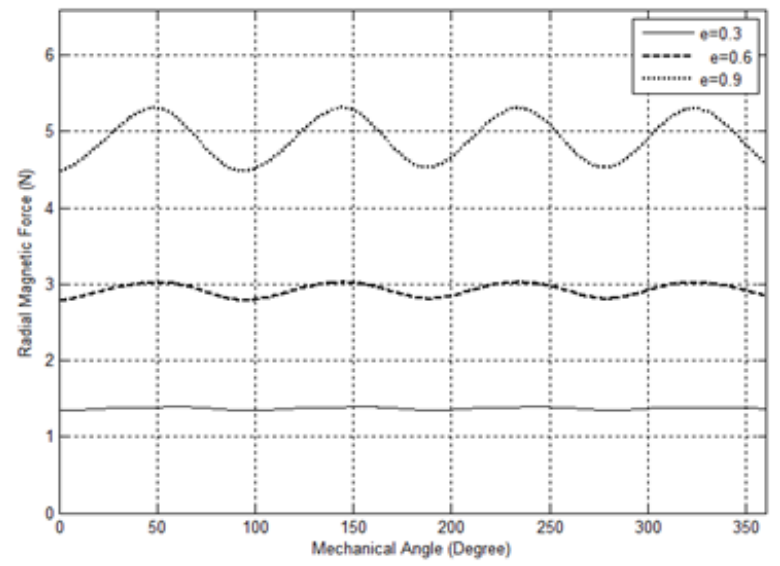

Fig. 6. Radial magnetic forces on the rotor for radial magnetization.

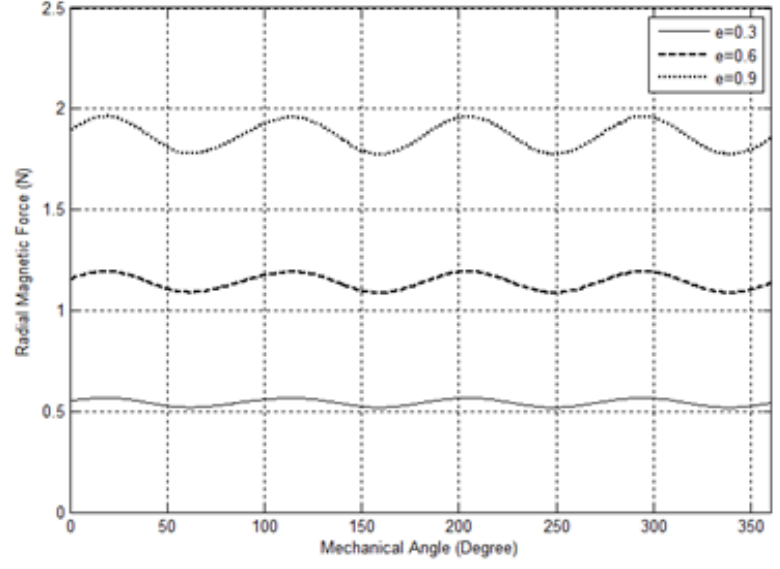

Fig. 7. Radial magnetic forces on the rotor for sinusoidal magnetization.

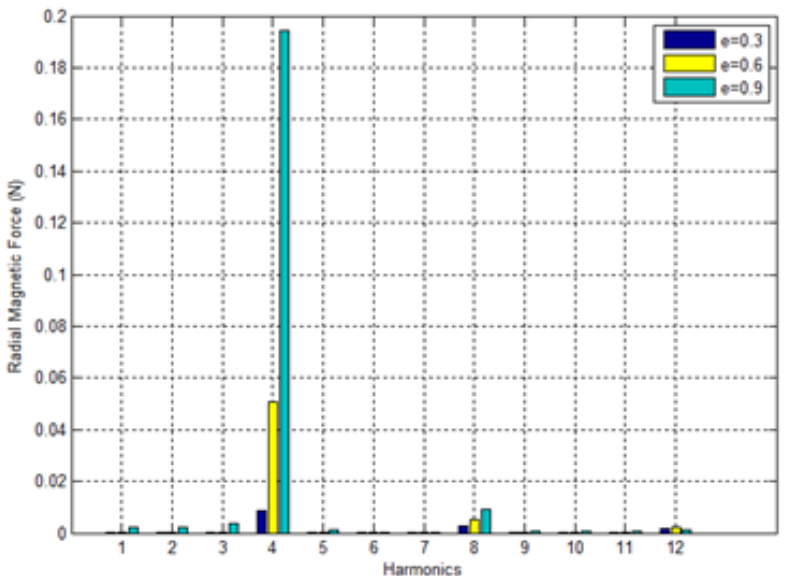

Fig. 8. Comparison of harmonic components of radial magnetic forces on the rotor for parallel magnetization.

The magnitude of the unbalanced magnetic force is known to be proportional to the amount of the eccentricity. Therefore, an efficient way of comparing two BLDC motors in terms of magnetic force characteristics is to assume the same amount of eccentricity in FEM calculation and compare the magnetic force profiles [3]. The average of these forces are calculated for each case in order to compare the effect of eccentricity ratio $e$ and different PM magnetization field patterns on radial magnetic forces simultaneously as shown in Figure 9. [8]:

The radial magnetic force on the rotor can be expressed as

$$
f_{r}=\frac{1}{2 \mu_{0}}\left(B_{r}^{2}-B_{\theta}^{2}\right)
$$

where $B_{r}$ and $B_{\theta}$ stands for radial and tangential component of magnetic flux density, respectively and $\mu_{0}$ is the permeability of the air. From (3) it can be seen that the radial magnetic force is proportional to the square of air gap magnetic flux density. 
It can be shown that PM generates the highest air gap flux density for radial magnetization and the lowest air gap flux density for sinusoidal magnetization. Therefore, radial magnetization gives the highest unbalanced magnetic force and sinusoidal magnetization offers the lowest unbalanced magnetic force on the rotor for all three different eccentricity ratios.

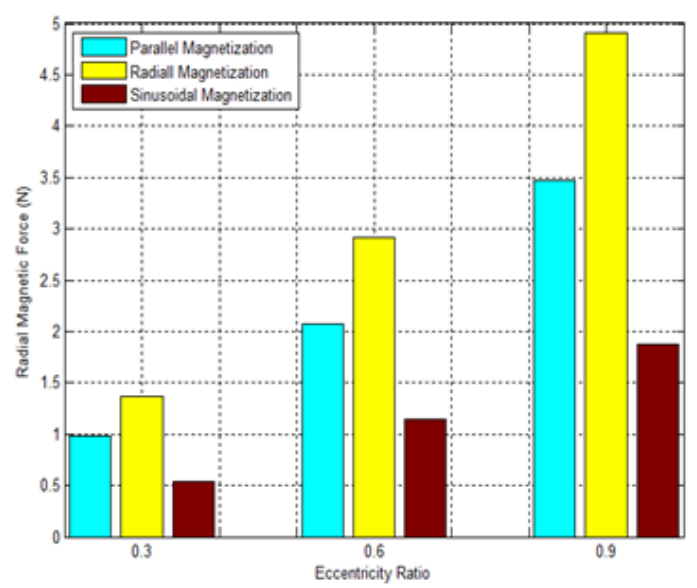

Fig. 9. Comparison of averages of radial magnetic forces on the rotor.

\section{DYNAMIC ECCENTRICITY ANALYSIS AND PESULTS}

In this section, dynamic eccentricity has been analyzed in the motor mentioned in section III and unbalanced magnetic forces have been compared for various rotor eccentricity conditions with some available ratios of $0.3,0.6$ and 0.9.

The calculation method of the radial magnetic force is similar to the one used in the case of static eccentricity explained in section IV. $X$ and $Y$ components and also amplitudes of unbalanced magnetic forces on the rotor for parallel magnetization with different eccentricity ratios, are shown in Figures 10-12.

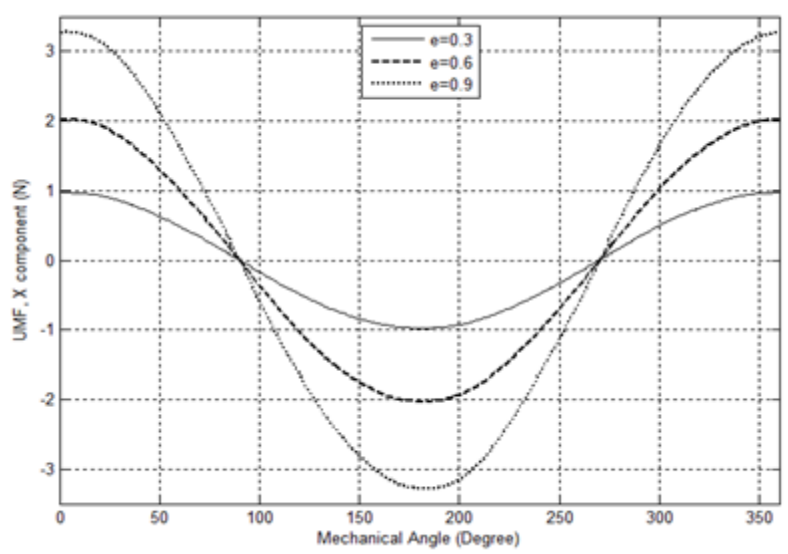

Fig. 10. X components of unbalanced magnetic forces on the rotor due to dynamic eccentricity of rotor for parallel magnetization.

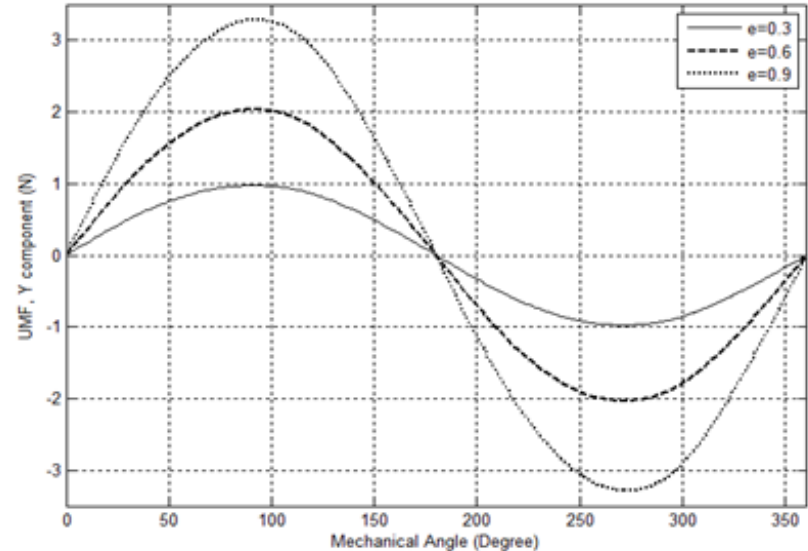

Fig. 11. Y components of unbalanced magnetic forces on the rotor due to dynamic eccentricity of rotor for parallel magnetization.

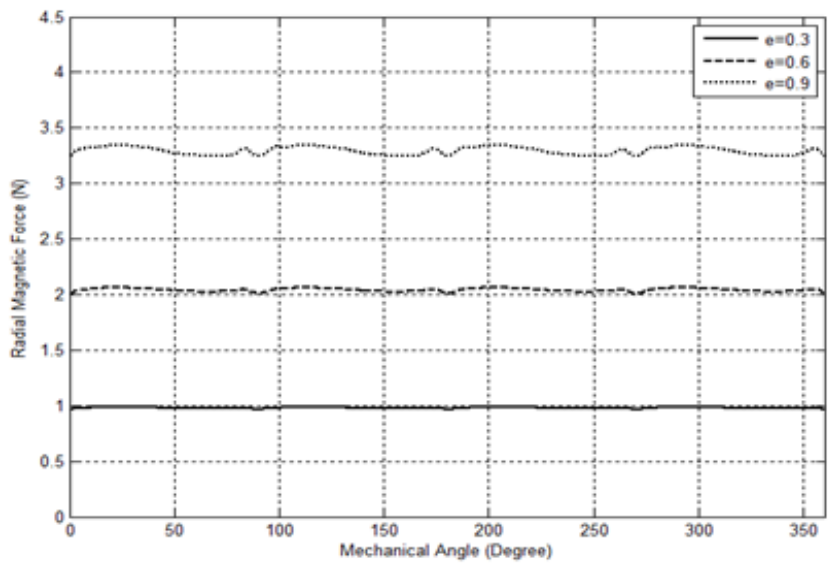

Fig. 12. Unbalanced magnetic forces on the rotor due to dynamic eccentricity of rotor for parallel magnetization.

As shown in Figures 10-11, $X$ and $Y$ components of unbalanced magnetic forces have negative and positive values in a complete revolution in dynamic eccentricity and this means that these forces revolve with rotor rotation. The magnitudes of the unbalanced magnetic forces on the rotor increase with the eccentricity ratio.

Harmonic components of the unbalanced magnetic force $X$ component on the rotor with eccentricity ratio of 0.3 are shown in Figure 13. As illustrated, it can be seen that in the dynamic eccentricity analysis, in addition to the first harmonic, 3th, 5th, 7th, 9th and 11th harmonic components are greater than other harmonic components. The reason is explained as follows.

In the case of dynamic eccentricity, the part of the rotor which is closer to the stator rotates with the rotor. Thus, a rotating radial magnetic force is generated and modulated by the slots. This force can be expressed as [9]:

$$
F_{r}=\sum_{i=0}^{n} f_{i} \sin \left(i l \omega t+\varphi_{i}\right)
$$


where $i, l$ and $\omega$ are the integer, slot number and rotational speed respectively.

$X$ and $Y$ components of the magnetic force can be expressed as follows:

$$
\begin{aligned}
& F_{X}=\cos (\omega t+\phi) \sum_{i=0}^{n} f \sin \left(i l \omega t+\varphi_{i}\right) \\
& =\frac{1}{2} \sum_{i=0}^{n} f_{i}\left[\sin \left((i l-1) \omega t+\varphi_{i}-\phi\right)\right. \\
& \left.+\sin \left((i l+1) \omega t+\varphi_{i}+\phi\right)\right] \\
& F_{Y}=\sin (\omega t+\phi) \sum_{i=0}^{n} f_{i} \sin \left(i l \omega t+\varphi_{i}\right) \\
& =\frac{1}{2} \sum_{i=0}^{n} f_{i}\left[\cos \left((i l-1) \omega t+\varphi_{i}-\phi\right)\right. \\
& \left.-\cos \left((i l+1) \omega t+\varphi_{i}+\phi\right)\right]
\end{aligned}
$$

where $\varphi$ is the angle between the stator rotation center and the rotor one. The above equations show that dynamic eccentricity leads to first harmonic $(i=0)$ and $i l \pm 1$ harmonic contents in the unbalanced magnetic force waveform.

The harmonic analysis of the unbalanced magnetic force is of great importance to the vibration analysis of the motor because large vibration will be generated when the natural frequencies of the motor coincide with or are close to the frequency of the electromagnetic force or its harmonic frequencies.

By comparing Figures 10 and 11, it can be deduced that the variation of $Y$ component of unbalanced magnetic force is similar to the $X$ component variation. Therefore, only the $X$ component has been investigated in this paper. On the other side, results obtained for the $X$ component of unbalanced magnetic force can be applied to the $Y$ component as well.

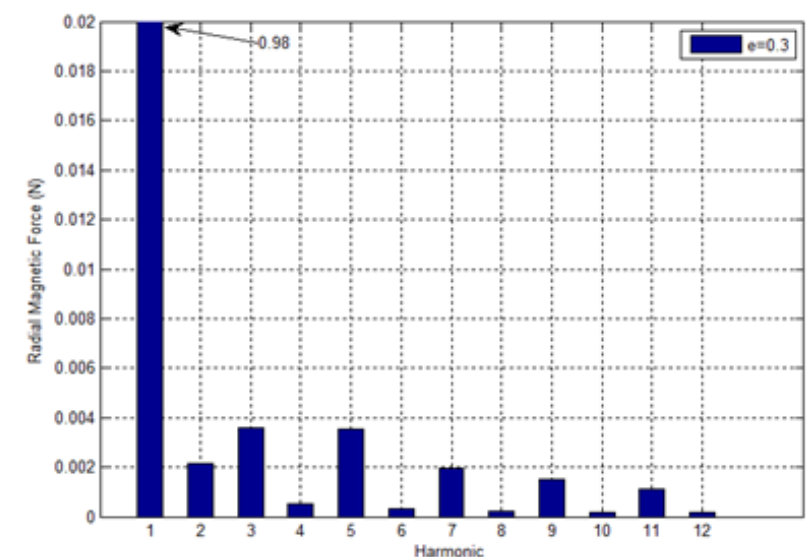

Fig. 13. Harmonics of $\mathrm{X}$ component of unbalanced magnetic forces on the rotor for parallel magnetization.

Finally, amplitudes of $X$ components of radial magnetic forces are calculated for each case in order to compare the effect of eccentricity ratio $e$ and different PM magnetization field patterns on radial magnetic forces simultaneously. The results are shown in Figure 14.

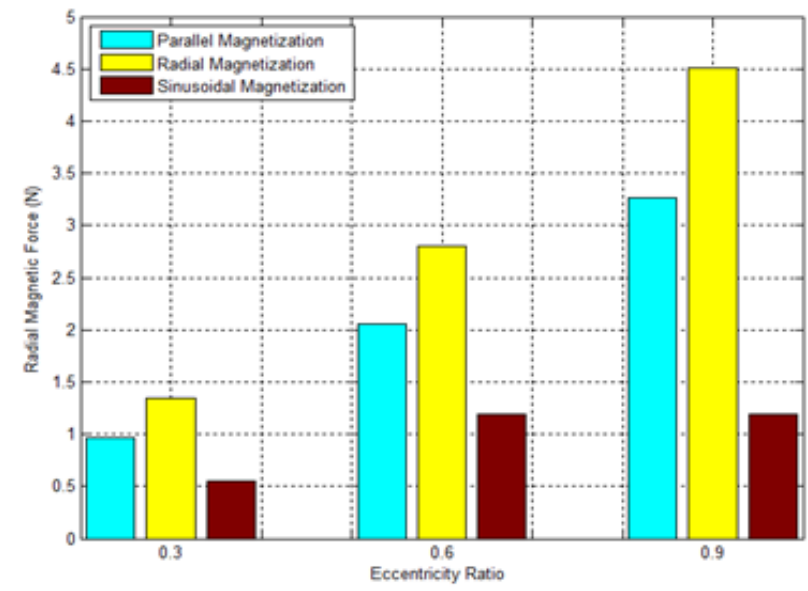

Fig. 14. Comparison of amplitudes of $\mathrm{X}$ component of radial magnetic forces on the rotor.

Considering (3), radial magnetic force is proportional to the square of air gap magnetic flux density. Therefore, with dynamic eccentricity radial magnetization produces the highest unbalanced magnetic force whereas sinusoidal magnetization generates the lowest unbalanced magnetic force on the rotor for all three different eccentricity ratios, as expected and shown in Figure 14.

\section{CONCLUSION}

Surface mounted PM motors may be less sensitive to rotor eccentricities than induction motors, and would be good candidates for applications where noise and vibration are significant but in the design of permanent magnet motors for high-precision applications, it is necessary to have a detailed analysis of the effect of rotor eccentricity. In this paper, static and dynamic eccentricities with eccentricity ratios of $0.3,0.6$ and 0.9 , are analyzed in a single phase BLDC motor. It can be seen from the simulation results that unbalanced magnetic forces acting on the rotor increase with the eccentricity ratio. Further, in the case of static eccentricity radial magnetic forces consist of harmonics which are multiples of the rotor pole numbers and in the case of dynamic eccentricity consist of the first harmonic and the harmonics which are multiples of slot numbers plus or minus one. It is clearly shown that the radial magnetization generates the highest unbalanced magnetic force and sinusoidal magnetization produces the lowest unbalanced magnetic force acting on the rotor in a BLDC motor.

\section{REFERENCES}

[1] H. S. Chen, M. C. Tsai "Effect of rotor eccentricity on electric parameters in a PM brushless motor with parallel winding connections", Journal of Applied Physics, Vol. 105, No. 7, pp. 07F121-07F121-3, 2009

[2] U. Kim, D. K. Lieu, "Magnetic field calculation in permanent magnet motors with rotor eccentricity: without slotting effect", IEEE Transactions on Magnetics, Vol. 34, No. 4, pp. 2243-2252, 1998 
[3] T. Yoon, "Magnetically induced vibration in a permanent-magnet brushless DC motor with symmetric pole-slot configuration", IEEE Transactions on Magnetics, Vol. 41, No. 6, pp. 2173-2179, 2005

[4] S. Rajagopalan, J. M. Aller, J. A. Restrepo, T. G. Halbetler, R.G. Harley, "Analytic-wavelet-ridge-based detection of dynamic eccentricity in brushless Direct Current BLDC motors functioning under dynamic operating conditions", IEEE Transactions on Industrial Electronics, Vol. 54, No. 3, pp. 1410-1419, 2007.

[5] S. Salon, K. Sivasubramaniam, L. T. Ergene, "The effect of asymmetry on torque in permanent magnet motors", IEMDC 2001, IEEE Electric Machines and Drives International Conference, Cambridge, USA, pp. 208-217, 2001

[6] Z. J. Liu, J. T. Li, M. A. Jabbar, "Prediction and analysis of magnetic forces in permanent magnet brushless dc motor with rotor eccentricity", Journal of Applied Physics, Vol. 99, No. 8, pp. 08R321- 08R321-3, 2006

[7] C. L. Chiu, Y. T. Chen, W. S. Jhang, "Properties of cogging torque, starting torque, and electrical circuits for the single-phase brushless DC motor", IEEE Transactions on Magnetics, Vol. 44, No. 10, pp. $2317-$ 2323, 2008

[8] K. T. Kim, S. M. Hwang, G. Y. Hwang, T. J. Kim, W. B. Jeong, C. U. $\mathrm{Kim}$, "Effect of rotor eccentricity on spindle vibration in magnetically symmetric and asymmetric BLDC motors", ISIE 2001, IEEE International Symposium on Industrial Electronics, Pusan, South Korea, Vol. 2, pp. 967-972, 2001

[9] C. I. Lee, G. H. Jang, "Experimental measurement and simulated verification of the unbalanced magnetic force in brushless DC motors", IEEE Transactions on Magnetics, Vol. 44, No. 11, pp. 4377-4380, 2008

\section{AUTHORS PROFILE}

Shahin Mahdiuon Rad received the B.Sc. degree in electrical engineering from the University of Zanjan, Iran, in 2008, and the M.Sc. degree, in 2011, in electrical engineering from the University of Tabriz, Iran. She is currently working toward the Ph.D. degree in the faculty of electrical engineering, Sahand University of Technology. Her research interests include control of electrical drives and electrical machines.

Seyed Reza Mousavi-Aghdam received his B.Sc. degree with first class honors in Electrical Power Engineering from Azarbaijan University of Tarbiat Moallem in 2009, Tabriz, and M.Sc. Degree from University of Tabriz with honor in 2011. He is currently working toward the Ph.D. degree in the University of Tabriz. His current research interests include design of electrical machines, electric drives and analysis of special machines.

Mohammad Reza Feyzi received his B.Sc. and M.Sc. in 1975 from the University of Tabriz in Iran with honors. He worked in the same University from 1975 to 1993 . He started his Ph.D. work at the University of Adelaide, Australia in 1993. Soon after his graduation, he rejoined the University of Tabriz. Currently, he is a professor in the same university. His research interests are finite element analysis, design and simulation of electrical machines and transformers.

Mohammad Bagher Bannae Sharifian studied Electrical Power Engineering at the University of Tabriz, Tabriz, Iran. He received the B.Sc. and M.Sc. degrees in 1989 and 1992 respectively from the University of Tabriz. In 1992 he joined the Electrical Engineering Department of the University of Tabriz as a lecturer. He received the Ph.D. degree in Electrical Engineering from the same University in 2000. In 2000 he rejoined the Electrical Power Department of the Faculty of Electrical and Computer Engineering of the same university as Assistant Professor. He is currently Professor of the mentioned Department. His research interests are in the areas of design, modeling and analysis of electrical machines, transformers, electric drives, liner electric motors, and electric and hybrid electric vehicle drives. 